\title{
Observation and Evaluation of Proton Diffusion in Porous Media by the pH-Imaging Microscope Using a Flat Semiconductor pH Sensor
}

\author{
Satoshi Nomura, ${ }^{* \dagger}$ Yan-Gou Yang, $* *$ Chihiro Inoue, ${ }^{* *}$ and Tadashi ChIDA** \\ * R\&D Center, Horiba Ltd., Kisshoinmiyanohigashimachi, Minami-ku, Kyoto 601-8510, Japan \\ **Department of Geoscience and Technology, Graduate School of Engineering, Tohoku University, \\ Sendai 980-8579, Japan
}

\begin{abstract}
The diffusion of protons in porous media was observed and evaluated using a pH-imaging microscope with a flat semiconductor. Small particles were packed on the sensing surface of the flat sensor, followed by the addition of a lactic acid droplet on the top of the packed particle layer. The $\mathrm{pH}$ distribution accompanied by the diffusion of lactic acid was visualized at the bottom of the layer using the flat sensor. The difference in the diffusion property was confirmed depending on the particle type. The effective diffusion coefficients of the lactic acid in some porous media were estimated by combining the experimental results and mathematical calculation.
\end{abstract}

(Received June 17, 2002; Accepted August 9, 2002)

\section{Introduction}

We have been developing a $\mathrm{pH}$-imaging microscope using a flat semiconductor $\mathrm{pH}$ sensor based on photocurrent characteristics of semiconductor silicon, which we call a Scanning Chemical Microscope. ${ }^{1-9}$ The sensor utilized in this microscope functions by light illumination to the opposite side of $\mathrm{pH}$ sensing. ${ }^{10}$ The sensor also functions as an array of multiple $\mathrm{pH}$ sensing parts by focussing and scanning the light illumination two dimensionally. The $\mathrm{pH}$ values obtained at each $\mathrm{pH}$ measurement point on the sensor are converted to a color scale, and the color scale of each $\mathrm{pH}$ value is displayed as $\mathrm{pH}$ images by the microscope. Taking advantage of the use of the sensor with a flat $\mathrm{pH}$-sensing surface and capability of multiple-point $\mathrm{pH}$ measurement, we have demonstrated a new type of surface characterization of solid samples using this microscope..$^{3-5}$ In this characterization, a thin agar film is placed on the flat sensor with the sample surface contacting the top of the agar film. Multiple-point $\mathrm{pH}$ measurements enable the $\mathrm{pH}$ distribution formed in the agar film to be imaged. Since the $\mathrm{pH}$ distribution in the agar film was formed by the contact of the sample to the agar film, the $\mathrm{pH}$ image reflects the surface chemical condition of the sample. Thus, the surface chemical information of the sample can indirectly be obtained. As an application of this unique surface analysis, we successfully imaged the surface of some biological samples, such as plant leaves and sliced human teeth. We also visualized the generation and expansion of electrochemically formed $\mathrm{pH}$ distributions while taking advantage of the use of a sensor with a flat $\mathrm{pH}$-sensing surface and the capability of multiple-point $\mathrm{pH}$ measurements in this microscope. ${ }^{2}$ Simple electrolysis was conducted in agar film formed on the flat sensor using a platinum anode and cathode.

$\doteqdot$ To whom correspondence should be addressed.

E-mail: nomura-s@ horiba.co.jp
The resulting lower and higher $\mathrm{pH}$ regions and their expansions were repeatedly visualized. Iwasaki et al. also conducted similar experiments in solutions with high viscosity, evaluating the diffusion coefficients of protons and hydroxide in solution with various viscosities. ${ }^{9}$

In this research, we successfully observed proton diffusion in a porous environment using our $\mathrm{pH}$-imaging microscope. The porous environment was formed by packing small particles on the flat sensor of the microscope with an electrolyte solution. Such an environment is regarded as an imitated soil system; thus, the observation of proton diffusion in the soil system was estimated in this research. The $\mathrm{pH}$ distribution formed in the bottom of the layer was repeatedly imaged after adding acid droplets on the top of the particle packed layer. The set of $\mathrm{pH}$ images obtained by repeated imaging showed the difference in the diffusion characteristics of acid inside the packed particle layer. ${ }^{11}$ In addition, these $\mathrm{pH}$ images allowed the effective diffusion coefficient of the acid, which was an important parameter in diffusion in porous media, to be calculated combined with a simple mathematical method.

Some other $\mathrm{pH}$-imaging methods have also been available with significant research results demonstrated so far. One method uses a scanning electrochemical microscope $(\text { SECM })^{12}$ with a miniaturized ion-selective potentiometric electrode as a sensing probe. As an application of this microscopy, a wide variety of reactions, such as corrosion, enzyme, and cell metabolism, were presented. The other is a fluorescence microscope combined with a $\mathrm{pH}$-sensitive fluorescent dye. As an application of this method, the $\mathrm{pH}$ distribution around the metal electrodes during water reduction and during corrosion was imaged with a relatively short time scale. ${ }^{12}$ These present methods have a higher spatial and time resolution than our presented $\mathrm{pH}$-imaging microscope. However, our $\mathrm{pH}$-imaging microscope requires neither scanning of the sensing probe in side the sample nor the addition of a $\mathrm{pH}$-sensitive fluorescent dye. This characteristic of our microscope is very important, 

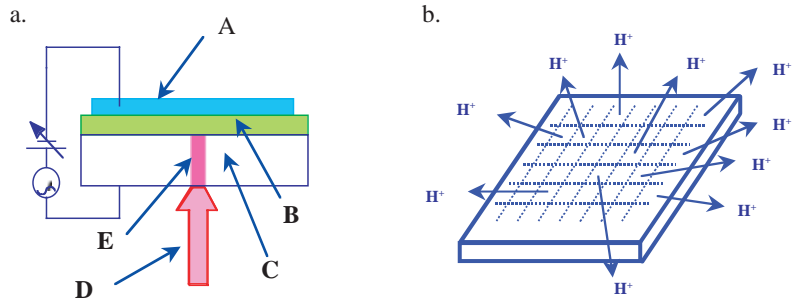

Fig. 1 a) Light addressable potentiometric sensor (LAPS): A, electrolyte; $\mathrm{B}$, insulator $\left(\mathrm{Si}_{3} \mathrm{~N}_{4} / \mathrm{SiO}_{2}\right) ; \mathrm{C}$, silicon; $\mathrm{D}$, light illumination; $\mathrm{E}$, photocurrent. b) pH-Imaging sensor. The sensor is functionally divided into multiple sensing parts based on the photocurrent characteristics of LAPS.

depending on the sample to be measured, even though the spatial and time resolution are lower. The use of a flat $\mathrm{pH}$ sensor was also advantageous in conducting the measurement using the packed particle layer. In this paper, sets of $\mathrm{pH}$ images and mathematical calculations are described, obtained as the result of proton diffusion in such porous media.

\section{Experimental}

\section{Instrument}

The pH-imaging microscope (SCHEM-100 Horiba Ltd, Japan) utilizes a silicon $\mathrm{pH}$ sensor with a flat sensing area of 2.5 $\mathrm{cm} \times 2.5 \mathrm{~cm}$. This sensor is based on a Light Addressable Potentiometric Sensor (LAPS) ${ }^{10}$ made up of a $\mathrm{Si}_{3} \mathrm{~N}_{4} / \mathrm{SiO}_{2} / \mathrm{Si}$ structure (Fig. 1). The proton-sensitive $\mathrm{Si}_{3} \mathrm{~N}_{4}$ surface was made to contact with an electrolyte, and a modulated light beam was illuminated from the Si side with a bias voltage applied between the electrolyte and the Si side. The characterization of the AC photocurrent induced by the modulated illumination from the $\mathrm{Si}$ side depends on the amount of protons at the $\mathrm{Si}_{3} \mathrm{~N}_{4}$ side, thus enabling $\mathrm{pH}$ measurements to be made. Since this sensor can only detect protons located in the illuminated region, the sensor can function as if it were divided into an array of small independent sensors by focusing and scanning the light illumination, though it is a single monolithic sensor. The modulation and scanning of light illumination, the application of a bias voltage and the data-acquisition function are all controlled by a computer. A computer connected to the microscope converts the $\mathrm{pH}$ values obtained by the sensor at each $\mathrm{pH}$-measurement point to a color scale, and then displays the color scale of each $\mathrm{pH}$ value as $\mathrm{pH}$ images. The spatial resolution and $\mathrm{pH}$ resolution of the imaging are $100 \mu \mathrm{m}$ and 0.1 $\mathrm{pH}$, respectively. ${ }^{1}$

\section{Measurement set-up and procedure}

A packed particle layer was formed by packing silica sand (425 - $600 \mu \mathrm{m}$ in diameter) or glass-beads particles $(400 \mu \mathrm{m}$ in diameter) on a pH-imaging sensor (Fig. 2). The area and height of the layer were $25 \mathrm{~mm} \times 25 \mathrm{~mm}$ and $4 \mathrm{~mm}$, respectively. A $0.1 \mathrm{M}$ potassium chloride solution was filled into the packed particles, followed by the addition of a $10 \mu \mathrm{l}$ lactic acid solution from the top surface of the layer. The $\mathrm{pH}$ distribution in a 20 $\mathrm{mm} \times 20 \mathrm{~mm}$ area was imaged with a $400 \mu \mathrm{m}$ spatial resolution. The time required for obtaining a single $\mathrm{pH}$ image was $120 \mathrm{~s}$. The imaging was repeated in $150 \mathrm{~s}$ intervals.

\section{Porosity of the particle packed layer}

The porosity $(\phi)$ of the packed particle layer was obtained by

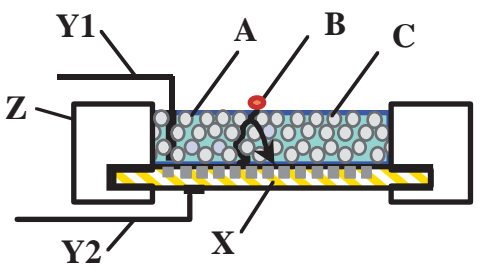

Fig. 2 Experimental set-up for observing proton transport in a porous environment. $\mathrm{X}, \mathrm{pH}$ imaging sensor; $\mathrm{Y} 1$ and $\mathrm{Y} 2$, electrodes for electric signals; $\mathrm{Z}$, sensor holder; A, potassium chloride solution; B, drop of acid solution; C, packed particles (glass beads, silica sand). The arrows indicate the movement of the acid solution.

poring deionized water in an acrylic pipe followed by packing of the particles in a pipe. ${ }^{14} \phi$ was calculated to be $38-39 \%$ according to

$$
\phi=\left\{\left(V_{\mathrm{w}}-\left(V_{\mathrm{t}}-V_{\mathrm{s}}\right)\right\} / V_{\mathrm{s}} \times 100,\right.
$$

where $V_{\mathrm{w}}$ is the actual volume of the deionized water, $V_{\mathrm{t}}$ the apparent volume of the deionized water when the particles were packed, and $V_{\mathrm{s}}$ the apparent volume of the packed particle layer, respectively.

\section{Results and Discussion}

The formation and expansion of a lower $\mathrm{pH}$ region was confirmed in a set of $\mathrm{pH}$ images obtained after the addition of lactic acid (Fig. 3). The shape of the lower $\mathrm{pH}$ region at each $\mathrm{pH}$ image was distorted compared to the round and uniform lower $\mathrm{pH}$ regions obtained by previous studies using agar film as a matrix. ${ }^{1,2}$ The distorted lower $\mathrm{pH}$ region shows that the particles were not packed completely uniformly, and that the protons could move faster in a relatively wider gap between each particle. The acid diffused faster in the case of glass beads, though the size of the particle was almost the same as that of silica sand. The faster proton diffusion in the glass beads layer can be explained by the difference in the tortuosity between the particles originating from the particle shape. The diffusion of the solute in porous media was estimated from the solute concentration measured at only one point of the layer combined with mathematical calculations in most present studies. ${ }^{6,10}$ Unlike these present researches, we enabled a clear visualization of proton diffusion in such a complicated environment by our $\mathrm{pH}$-imaging microscope.

The diffusion of the acid was evaluated quantitatively based on the $\mathrm{pH}$ images shown in Fig. 3. The effective diffusion coefficient $\left(D_{\mathrm{e}}\right)$ of the acid was obtained by the following procedure. First, the ratio of the detected total proton amount $\left(Q_{\mathrm{t}}\right)$ over the induced proton amount $\left(Q_{\mathrm{m}}\right)$ was calculated based on the obtained $\mathrm{pH}$ images. $Q_{\mathrm{t}}$ was calculated based on

$$
Q_{\mathrm{t}}=\sum_{i=1, j=1}^{i_{\mathrm{m}} j_{\mathrm{m}}} c_{i, j, 1} x_{c} y_{c} H \phi \rho
$$

where $c_{i, j}$ is the proton concentration obtained by the $\mathrm{pH}$ value at each measurement point; $i_{\mathrm{m}}$ and $j_{\mathrm{m}}$ are the numbers of measuring points $\left(i_{\mathrm{m}}=j_{\mathrm{m}}=50\right) ; x_{\mathrm{c}}$, and $y_{\mathrm{c}}$ are the resolutions of the measuring points $\left(x_{\mathrm{c}}=y_{\mathrm{c}}=400 \mu \mathrm{m}\right), H$ is the height of the packed-particle layer and $\rho$ is the density of the acid, respectively. The ratio $\left(Q_{\mathrm{t}} / Q_{\text {in }}\right)$ is plotted in Fig. 4 . Then, the ratio $\left(Q_{\mathrm{t}} / Q_{\text {in }}\right)$ was theoretically calculated using the three- 
a. 0
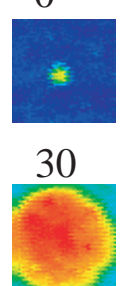

b. 0

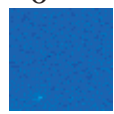

30

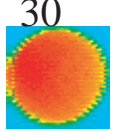

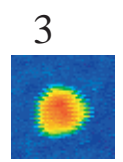
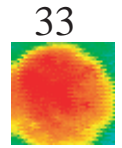

3
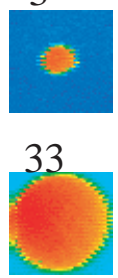
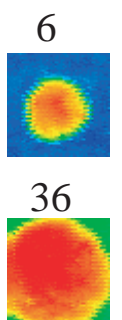

6

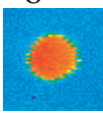

36

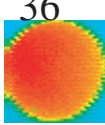

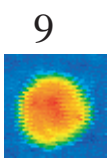

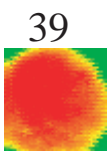

9

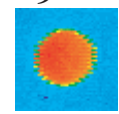

39

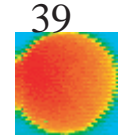

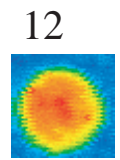

42

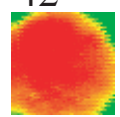

12

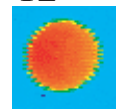

42

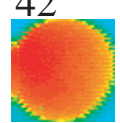

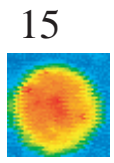

45

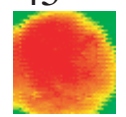

15

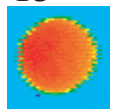

45

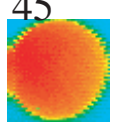

18

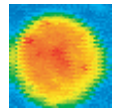

21

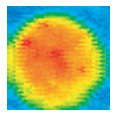

24

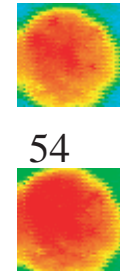

27

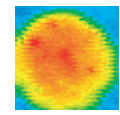

$\mathrm{pH}$
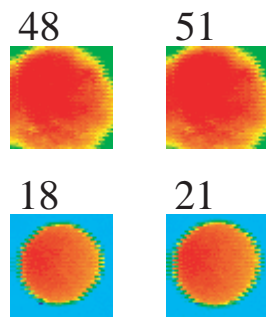

21

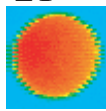

24
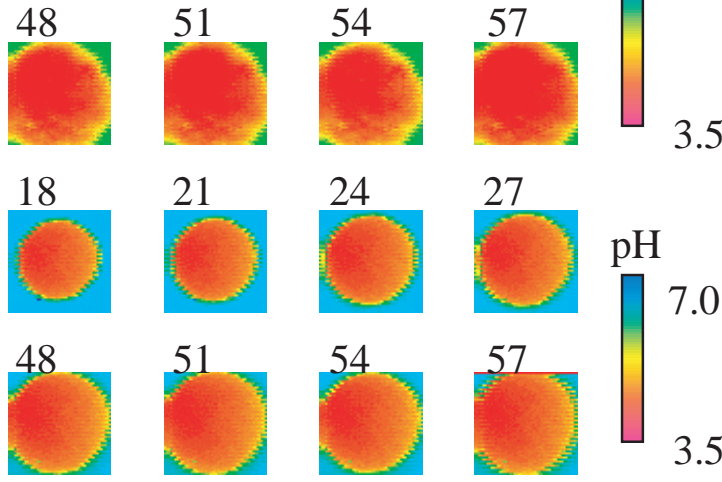

Fig. $3 \mathrm{pH}$ distributions formed in a packed-particle bead and agar gel. a) Glass beads (400 $\mu \mathrm{m})$. b) Silica sand (425 - 600 $\mu \mathrm{m})$; image size, $20.0 \mathrm{~mm} \times 20.0 \mathrm{~mm}$; measured points, $40 \times 40$. The number at each image shows the time imaging started.

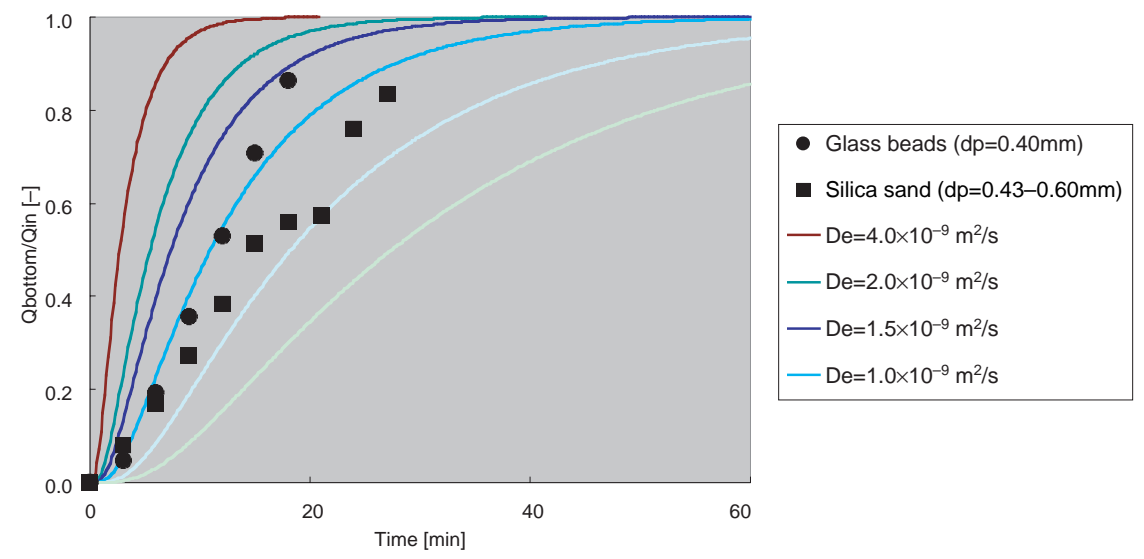

Fig. 4 Dependence of $Q_{t} / Q_{\text {in }}$ on the particle types measured in a packed bead of glass beads or silica sands.

dimensional proton concentration changes which could be expected according to the following diffusion equation using the effective diffusion coefficient $\left(\mathrm{m}^{2} \mathrm{~s}^{-1}\right)$ of the lactic acid $\left(D_{\mathrm{e}}\right)$ in a porous environment:

$$
\frac{\partial c}{\partial t}=D_{\mathrm{e}}\left(\frac{\partial^{2} c}{\partial x^{2}}+\frac{\partial^{2} c}{\partial y^{2}}+\frac{\partial^{2} c}{\partial z^{2}}\right)
$$

Here, $c$ is the concentration of protons $\left(\mathrm{kg} \mathrm{m}^{-3}\right), t$ is time (s), and, $x$ and $y$ are the axes (m). The theoretical $Q_{\mathrm{t}} / Q_{\mathrm{m}}$ value could be calculated in the case of various $D_{\mathrm{e}}$ values, and is shown in Fig. 4. By curve fitting between the experimental and theoretical $Q_{\mathrm{t}} / Q_{\mathrm{m}}$ values, the $D_{\mathrm{e}}$ under each experimental condition could be estimated (listed in Table 1). Considerable accordance between the $D_{\mathrm{e}}$ and $D$ values was confirmed in the case of both glass beads and silica sand, though the diffusion of the acid was influenced by the surface conditions, such as the wettability, shape and surface area of the packed particles in the packed-particle layer. The diffusion coefficients of some other organic acids are also listed in Table 1.

The diffusion of chemical substances occurs not only in uniform media, but also in more complicated environments. ${ }^{17-20}$ For example, chemical pollutants diffuse in a subsurface
Table 1 Simulated diffusion coefficient of lactic acid in a packed-particle layer $\left(10^{-9} \mathrm{~m}^{2} / \mathrm{s}\right)$

\begin{tabular}{|c|c|c|}
\hline & \multicolumn{2}{|c|}{ Particle type and size } \\
\hline & Glass beads & Silica sand \\
\hline$D_{\mathrm{e}^{\mathrm{a}}}$ & 1.5 & 1.0 \\
\hline$D^{\mathrm{b}}$ & \multicolumn{2}{|c|}{1.10} \\
\hline$D(\mathrm{HCOOH})^{\mathrm{c}}$ & \multicolumn{2}{|c|}{1.42} \\
\hline$D\left(\mathrm{CH}_{3} \mathrm{COOH}\right)^{\mathrm{d}}$ & \multicolumn{2}{|c|}{1.08} \\
\hline
\end{tabular}

a. $D_{\mathrm{e}}$ : simulated effective diffusion coefficient of lactic acid in packed-particle layer (room temperature).

b. $D$ : diffusion coefficient of lactic acid in aqueous solution $\left(25^{\circ} \mathrm{C}\right){ }^{15}$

c. $D(\mathrm{HCOOH})$ : diffusion coefficient of $\mathrm{HCOOH}$ in aqueous solution $\left(25^{\circ} \mathrm{C}\right) .^{16}$

d. $D\left(\mathrm{CH}_{3} \mathrm{COOH}\right)$ : diffusion coefficient of $\mathrm{CH}_{3} \mathrm{OOH}$ in aqueous solution $\left(25^{\circ} \mathrm{C}\right) .{ }^{16}$

environment, which is regarded as a porous medium consisting of mineral, solution and gas phases. These phases intact with each other. The effective diffusion coefficient of the solute is 
one of the most important parameters in order to assume the movement of solute in the porous media. Our method described in this paper will be applied to evaluate the effective diffusion coefficients of acids in various particle types, enabling us to contribute to the understanding of mass transfer in porous media. As a promising feature of our $\mathrm{pH}$-imaging analysis described in this paper, applicability to a laboratory-scale evaluation of the bioremediation process ${ }^{21}$ or a microbiologicalenhanced oil recovery process ${ }^{22,23}$ is expected. In order to conduct these processes efficiently, understanding both nutrient diffusion and the behavior of microbiology in a porous environment is important. A preliminary experiment using Lactobacillus casei is now underway.

\section{Conclusion}

We have proposed a new method to evaluate proton diffusion in a packed particle layer using $\mathrm{pH}$-imaging analysis. The dependence of proton transport on the particle type was clearly imaged qualitatively. As a quantitative evaluation, an effective diffusion coefficient of lactic acid was obtained. The established method can be used for understanding mass transport in soil systems under laboratory conditions, thus contributing to fundamental research involving the transport of chemicals in soil systems.

\section{Acknowledgements}

The authors thank Professor Hiroshi Iwasaki and Dr. Tatsuo Yoshinobu for stimulating discussion regarding the $\mathrm{pH}$-imaging sensor. This study was supported in part by a Grant-in-Aid for Science Research (11450394) from the Ministry of Education, Culture, Sports, Science and Technology of Japan.

\section{References}

1. S. Nomura, M. Nakao, T. Nakanishi, S. Takamatsu, and K. Tomita, Anal. Chem., 1997, 69, 977.

2. T. Yoshinobu, H. Iwasaki, S. Nomura, M. Nakao, T. Nakanishi, S. Takamatsu, and K. Tomita, Jpn. J. Appl. Phys., 1998, 37, L353.

3. Y. Kitasako, T. Nikaido, J. Tagami, K. Ikeda, K. Mitsunari, and S. Nomura, Bunseki Kagaku, 2000, 2, 325.

4. S. Nomura, K. Mitsunari, M. Nakao, Y. Kohno, and R. Matsuki, G.I.T IMAGING \& MICROSCOPY, 2000, 2, 59.

5. Y. Kitasako, N. Hiraishi, M. Nakajima, T. Nikaido, J. Tagami, and S. Nomura, Jpn. J. Conserv. Dent., 2001, 44, 56.

6. M. Nakao, T. Yoshinobu, and H. Iwasaki, Jpn. J. Appl. Phys., 1994, 33, L394.

7. M. Nakao, T. Yoshinobu, and H. Iwasaki, Sens. Actuators, 1994, B20, 119.

8. M. Nakao, S. Inoue, R. Oishi, T. Yoshinobu, and H. Iwasaki, "Tech. Digest, 8th. Conf. Solid-state Sensors \& Actuators (Transducers '95)", 451.

9. T. Yoshinobu, A. B. M. Ismail, T. Harada, and H. Iwasaki, Jpn. J. Appl. Phys., 2000, 39, L400.

10. D. J. Hafeman, J. W. Parce, and H. M. McConnell, Science, 1988, 240, 1182.

11. S. Nomura, M. Nakao, S. Takamatsu, Y. G. Yang, C. Inoue, and T. Chida., Bunseki Kagaku, 1999, 48, 763.

12. B. R. Horrocks, M. V. Mirkin, D. T. Pierce, A. J. Bard, G. Nagy, and K. Toth, Anal. Chem., 1993, 65, 1213.

13. R. C. Engstrom, S. Chaffari, and H. Qu, Anal. Chem., 1992, $64,2525$.

14. J. Bear and J. M. Buchlin, "Modeling and applications of transport phenomena in porous media", 1987, Kluwer Academic Publishers, 344 - 345.

15. T. J. Chresand, B. E. Dale, and S. L. Hanson, Biotechnol. Bioeng., 1988, 32, 1029.

16. "Chemical data table", 1993, Maruzen, Tokyo, II-93.

17. J. Brakel and P. M. Heertjes, Int. J. Heat Mass Transfer, 1974, 17, 1093.

18. J. A. Ochoa-Tapia, P. Stroeve, and S. Whitaker, Chem. Eng. Sci., 1991, 46, 177.

19. M. Victor, M. Lobo, and A. J. M. Valente, Intern. J. Polymeric. Matter., 1994, 25, 139.

20. Q. Hu and M. L. Brusseau, Water Resources Res., 1995 , 31, 1637.

21. R. M. Maier, I. L. Pepper, and C. P. Gerba, "Environmental Microbiology", 2000, Academic Press, 61.

22. H. Mulder, A. M. Breure, and W. H. Rulkens, Chemosphere, 2001, 42, 285.

23. Y. G. Yang, Y. Niibori, C. Inoue, and T. Chida, Proceedings of the IASTED International Conference on Applied Modeling and Simulation, 1999. 